\title{
Elementos diagnósticos en medicina interna 2003-2010
}

\author{
Jordi Delás, Cesar Morcillo, Gemma Martín, Adrià Arboix, Enric Gil de Bernabé, Olga Parra
}

Introducción. Desde el curso 2003-2004 impartimos en la Facultad de Medicina de la Universitat de Barcelona la asignatura optativa semipresencial 'Elementos diagnósticos en medicina interna', en la que se aborda el diagnóstico a través de análisis de laboratorio, pruebas funcionales, de imagen y electrocardiografía.

Materiales y métodos. Tras las sesiones presenciales, el material docente se deposita en la página web o campus virtual de la asignatura, a partir del cual los estudiantes responden a seis cuestionarios.

Resultados. En el curso 2008-2009 se matricularon 61 alumnos de cuarto a sexto curso. Veintisiete alumnos (44,26\%) respondieron correctamente a más de la mitad de las preguntas, el 31,15\% no alcanzó esta cifra y el 24,59\% no completó el programa de evaluaciones. Las puntuaciones más altas se han dado de las pruebas funcionales respiratorias, y las más bajas, en la evaluación de la radiografía de tórax.

Conclusiones. No es fácil la evaluación de competencias clínicas. El coste de realización de las pruebas y la resistencia de los estudiantes a ser evaluados quedan soslayados en esta experiencia, donde los estudiantes se matriculan voluntariamente. La sencillez del proyecto permitiría evaluar en competencias clínicas a todos los estudiantes de la facultad en los cursos clínicos cuando aún es posible la formación. Una vez comprobado que los estudiantes tienen una buena aceptación del sistema propuesto, se podría diseñar un sistema de acceso a créditos docentes y un plan de formación específica en las materias no superadas.

Palabras clave. Análisis de laboratorio. Diagnóstico por la imagen. Educación médica. Electrocardiograma. Habilidades clínicas.

\section{Diagnostics elements in internal medicine 2003-2010}

Introduction. From the course 2003-2004, we give in the Faculty of Medicine of the University of Barcelona a matter called Diagnostics Elements in Internal Medicine, for the diagnostic through analysis of laboratory, functional proofs, of image and electrocardiogram.

Materials and methods. All the educational material is deposited in the page web of the topic and through it the students answer the 6 questionnaires.

Results. In the course 2008-2009, 61 students have choose the topic. 27 students (44,26\%) answered correctly more than $50 \%$ of the questions. $31,15 \%$ did not achieve this figure and $24,59 \%$ did not complete the program of evaluations. The highest punctuations have given in respiratory functional proofs and the lowest in the evaluation of the thoracic X-ray.

Conclusions. It's not easy the evaluation of clinical skills. The cost of realization of the proofs and the resistance of the students to be evaluated remain skewed in this experience where the students participate voluntarily. The simplicity of the project would allow evaluating in clinical skills all the students of the faculty in the clinical courses period when it still is possible the training. The students have a good acceptance of the system proposed, and it could design a system of access to educational credits and a plan of specific training in the no surpassed matters.

Key words. Analysis of laboratory. Clinical skills. Electrocardiogram. Imaging diagnosis. Medical education.

\section{Introducción}

Es posible que los estudiantes de medicina acaben sus estudios sin dominar competencias clínicas básicas que mayoritariamente han de adquirirse durante la estancia en las áreas clínicas de hospitales o centros de atención primaria, pero que habitualmente no se imparten ni se evalúan de forma sistemática. Son competencias del tipo de interpretación de análisis de laboratorio, radiografías de tórax y abdomen, electrocardiogramas o pruebas funcionales respiratorias.
Hospital Universitari del Sagrat Cor (J. Delás, A. Arboix, E. Gil de Bernabé, O. Parra). CIMA (C. Morcillo, E. Gil de Bernabé). Hospital del Mar (G. Martín). Barcelona, España.

Correspondencia: Dr. Jordi Delás. Servicio de Medicina Interna. Hospital Universitari del Sagrat Cor. Viladomat, 288. E-08029 Barcelona.

E-mail:

jdelas@ub.edu

Conflicto de intereses: No declarado.

Conflict of interests: None declared.

(c) 2012 Educación Médica 
En este sentido, desde el curso 2003-2004 proponemos una asignatura optativa, denominada 'Elementos diagnósticos en medicina interna, en la que intentamos sistematizar docencia y evaluación clínica.

La asignatura tiene una duración de tres meses, de octubre a diciembre, con una sesión semanal de dos horas de duración, los viernes por la mañana, en la que se abordan los principales motivos de consulta, elementos diagnósticos habituales, y protocolos y consensos en relación a las competencias planteadas.

El material elaborado por los profesores se deposita en la página web -o campus virtual- de la asignatura y la evaluación se realiza de forma no presencial.

En todas las ediciones se han cubierto el total de plazas ofertadas, desde 20 en la primera edición hasta los más de 50 estudiantes en la actualidad.

Para una mejor exposición de esta herramienta docente presentamos la evaluación del curso 20082009, de acuerdo con el principio de que una de las grandes cuestiones e incluso responsabilidades en docencia es garantizar que el estudiante acceda a los adecuados conocimientos en cada disciplina [1].

\section{Materiales y métodos}

Hemos evaluado la capacidad de interpretación de análisis de laboratorio, radiografía de tórax, pruebas funcionales respiratorias, electrocardiograma y radiografías de abdomen de los estudiantes de medicina de cuarto, quinto y sexto curso de la Facultad de Medicina de la Universitat de Barcelona, matriculados en el curso 2008-2009 en la asignatura optativa 'Elementos diagnósticos en medicina interna'.

El equipo docente está formado por dos internistas, un neurólogo, una neumóloga, un cirujano general, un cirujano torácico y una dermatóloga.

Después de las sesiones presenciales, los profesores depositan el material docente en la página web o campus virtual de la asignatura. Los estudiantes han de responder a seis cuestionarios propuestos sucesivamente en el plazo de 10 días a través del campus virtual de la asignatura [2], basado en el paquete de software Moodle para la creación de cursos y sitios web basados en Internet [3].

Se permite un único intento, desde el ordenador que cada alumno escoja, sin otro límite que los plazos de entrega. Pasado el día y hora señalados, el programa no permite cumplimentar el cuestionario.

La evaluación se basa en la respuesta a preguntas de elección múltiple con más de una posibilidad verdadera y puntuación negativa de las respuestas erróneas. El programa cambia el orden de las preguntas y, dentro de las preguntas, el orden de las res- puestas en cada intento. La corrección es automática a partir del programa informático.

Se registra el número de preguntas planteadas en cada evaluación, el total de respuestas posibles, el número de alumnos que las ha respondido y el porcentaje de alumnos que ha superado cada pregunta planteada.

Se identifican las respuestas falsas que han escogido menos del $5 \%$ de los estudiantes y los resultados obtenidos por los alumnos.

\section{Resultados}

En el curso 2008-2009 se matricularon en la asignatura 61 alumnos de cuarto, quinto y sexto curso. Respondieron a las evaluaciones un mínimo de 51 alumnos y un máximo de 53. Las preguntas de cada evaluación oscilaron desde nueve en la evaluación de la radiografía de tórax hasta 20 en la evaluación de análisis de laboratorio. Cada evaluación supuso entre 50 y 100 propuestas no excluyentes entre sí, con un valor promedio de 71 propuestas por evaluación y un total de más de 350 propuestas no excluyentes (Tabla I).

En cada ejercicio se calculó el porcentaje de estudiantes que contestaron adecuadamente cada pregunta y el valor promedio (Tabla I). El valor más alto se dio en la evaluación de las pruebas funcionales respiratorias, y el más bajo, en la evaluación de la radiografía de tórax.

Una vez transcurrido el plazo de entrega de todos los ejercicios, 27 alumnos (44,26\%) de los 61 matriculados respondieron correctamente a más del $50 \%$ de las preguntas en las cinco evaluaciones. El $31,15 \%$ no alcanzó está cifra y el $24,59 \%$ no completó el programa de evaluaciones (Tabla II).

\section{Discusión}

En los objetivos de los estudios de medicina de las diferentes facultades se suelen describir las competencias que debe adquirir el estudiante durante su periodo de formación. Pero no es fácil su evaluación, en especial cuando se trata de competencias clínicas que se han abordado en distintas asignaturas y diferentes estancias clínicas en centros sanitarios [4]. Existen numerosos proyectos de evaluación clínica de los alumnos y, de hecho, hemos realizado alguno en nuestro propio hospital [5,6]. Los principales problemas residen en el coste de realización de las pruebas y la resistencia de los estudiantes a ser evaluados. Ambos aspectos quedan soslayados 
Tabla I. Resultados generales.

\begin{tabular}{lcccccc}
\hline & Análisis & $\begin{array}{c}\text { Radiografías } \\
\text { de tórax }\end{array}$ & $\begin{array}{c}\text { Pruebas funcionales } \\
\text { y gasometría }\end{array}$ & $\begin{array}{c}\text { Electro- } \\
\text { cardiogramas }\end{array}$ & $\begin{array}{c}\text { Radiografías del } \\
\text { aparato digestivo }\end{array}$ & Promedio \\
\hline $\begin{array}{l}\text { Alumnos que han } \\
\text { realizado la evaluación }\end{array}$ & 52 & 53 & 53 & 51 & 53 & 52,4 \\
\hline $\begin{array}{l}\text { Total de preguntas } \\
\text { Total de respuestas posibles }\end{array}$ & 100 & 60 & 50 & 95 & 50 & 73,6 \\
\hline $\begin{array}{l}\text { Respuestas falsas que han } \\
\text { escogido menos del } 5 \%\end{array}$ & $10(10 \%)$ & $15(25 \%)$ & $9(18 \%)$ & $27(54 \%)$ & $12(24 \%)$ & $26 \%$ \\
$\begin{array}{l}\text { Preguntas adecuadamente } \\
\text { contestadas a }\end{array}$ & $62 \%$ & $54 \%$ & $72 \%$ & $59 \%$ & $60 \%$ & $61 \%$ \\
\hline
\end{tabular}

a Valor promedio entre los estudiantes que han realizado la evaluación.

en esta experiencia, donde los estudiantes se matriculan voluntariamente en una asignatura que implica evaluación de material (análisis, radiografías, electrocardiogramas, pruebas funcionales) que habitualmente se utiliza en la clínica. No se exige la asistencia a clase. El alumno tiene la opción de superar las evaluaciones sin haber acudido ningún día a clase o puede asistir a las experiencias y explicaciones de los profesores en las clases presenciales. Habitualmente acuden de 15 a 20 alumnos.

En la evaluación empleamos preguntas de elección múltiple $[7,8]$, según una opción que ofrece el campus virtual de la Universitat de Barcelona, que sigue el paquete de software Moodle para la creación de cursos y sitios web. Permite la evaluación por ordenador de un gran número de estudiantes en un corto periodo [9]. A este respecto, es interesante destacar el progresivo interés sobre cómo realizar evaluaciones por parte de diferentes autores [10].

En la evaluación utilizamos la modalidad de más de una respuesta válida porque consideramos que es la fórmula que más se acerca a la realidad clínica, donde no hay una única verdadera posibilidad. En este contexto, las respuestas erróneas obligatoriamente han de puntuarse de forma negativa, para evitar que pueda conseguirse la máxima puntuación en la pregunta marcando todas las opciones posibles.

Es una evaluación exigente, que penaliza de forma importante la nota final, pero nos parece aceptable en una evaluación no presencial.

No nos preocupa que los estudiantes puedan recurrir a otras personas para la solución de los cuestionarios. De hecho es una posibilidad habitual en cualquier evaluación no presencial, como la realiza-
Tabla II. Resultados globales $(n=61)$.

\begin{tabular}{lcc}
\hline & $n$ & $\%$ \\
\hline Han superado las cinco evaluaciones & 27 & 44,26 \\
\hline No han superado los ejercicios & 15 & 24,59 \\
\hline $\begin{array}{l}\text { No han realizado los ejercicios } \\
\text { No han realizado los ejercicios y no } \\
\text { han superado alguno de los ejercicios }\end{array}$ & 15 & 24,59 \\
\hline
\end{tabular}

ción de un trabajo académico fuera de la observación del profesor. Incluso nos parece positivo que los estudiantes recurran a otros compañeros -en lo que podría ser un ejercicio de enseñanza entre pares o iguales- o a médicos en ejercicio. De hecho, consultar constituye una práctica habitual del clínico.

En anteriores ediciones comprobamos que más del $90 \%$ de los estudiantes enviaban los exámenes desde ordenadores diferentes. Es decir, no utilizaban los ordenadores de la facultad, lo que hubiera podido ser un elemento de confusión. De ello deducimos que los estudiantes no respondían los cuestionarios en grupo.

El diferente número de preguntas de cada evaluación se relaciona con los objetivos perseguidos en cada caso. Intentamos que los cuestionarios sean cortos [11], y lo facilita el hecho de que pueda haber más de una respuesta verdadera.

Las más de 350 propuestas sobre elementos diagnósticos ofrecen una importante información de los 
conocimientos de los alumnos. El porcentaje de preguntas adecuadamente contestadas informa del conocimiento del grupo sobre el tema evaluado. Es una información valiosa que permite detectar las materias en las que los estudiantes tienen menos conocimientos, así como analizar la trayectoria en la formación de los alumnos en las materias con mejores resultados para intentar aplicarla a otros campos. Ello puede llevar a aumentar el grado de exigencia en temas adecuadamente respondidos en un mayor porcentaje (pruebas funcionales respiratorias) o mejorar el programa de formación previa a las evaluaciones y, especialmente, comprobar si se ajusta la información impartida con las cuestiones a evaluar. En sucesivas ediciones pueden sustituirse elementos que se consideran ya conocidos por la mayoría de alumnos y sustituirlos por otros.

Unos mejores resultados pueden derivar también de una menor dificultad de las preguntas. Para aproximarnos a esta cuestión, hemos calculado el porcentaje de respuestas falsas excesivamente obvias y que, por tanto, han escogido menos del $5 \%$ de los alumnos. Habitualmente las sustituimos en sucesivas ediciones a fin de descartar que unos mejores resultados se deban a preguntas más sencillas o menos verosímiles.

Al considerar el promedio de estudiantes que han respondido adecuadamente las diferentes evaluaciones observamos que los peores resultados se han obtenido en la evaluación de electrocardiogramas (59\%) y radiografías de tórax (54\%). Sin embargo, ambas evaluaciones se han dado con índices altos de respuestas falsas escasamente seleccionadas por los estudiantes, lo que podría plantear que los resultados más bajos probablemente se deban a los conocimientos de los alumnos y no a la facilidad de las preguntas (Tabla I).

El 25\% de los estudiantes no completó la totalidad de los ejercicios en el plazo propuesto, habitualmente 10 días. Al tratarse de una asignatura optativa puede ser que no haya respondido a sus expectativas y la hayan abandonado. La otra posibilidad es que no hayan sido capaces de seguir el ritmo de una evaluación continuada. En ambos casos se trata de mejorar la información previa del alumno, para que no se produzca un porcentaje tan elevado de abandonos.

Sólo el 44,26\% superó las cinco evaluaciones. Al $31,15 \%$ restante se le ofreció la posibilidad de repetir las evaluaciones tras un breve periodo de formación. Es decir, al detectarse deficiencia en algunas de las competencias evaluadas se les propuso formarse específicamente a partir del material impartido por los profesores y repetir la evaluación.
Puede ser discutible que en una evaluación no presencial el punto de corte se sitúe en el $50 \%$. O lo que es lo mismo, que el 50\% suponga dominar dichas competencias. Cabe la posibilidad en sucesivas ediciones de elevar el citado punto de corte.

No es un proyecto dirigido a la excelencia o a detectar los estudiantes con mejor formación en las materias planteadas. Es un proyecto de mínimos destinado a garantizar que, al final de la asignatura, los alumnos tengan una formación suficiente para afrontar la interpretación de determinados elementos diagnósticos. Por ello, al alumno que no ha superado alguna de las seis evaluaciones propuestas se le ofrece prepararse de nuevo con el material docente de la asignatura y realizar una nueva evaluación al cabo de pocas semanas.

La sencillez del proyecto permitiría evaluar en competencias clínicas a todos los estudiantes de la facultad en la segunda parte de la carrera y cuando todavía es posible la formación. Resultaría preciso consensuar los elementos a evaluar, contar con un equipo de evaluación y que los estudiantes realicen los ejercicios de forma no presencial. Ya hemos comprobado que los estudiantes tienen una buena aceptación del sistema propuesto. Además de poder acceder a créditos, el estudiante podría recibir un plan de formación focalizado en las materias no superadas.

Bibliografía

1. Palmer EJ, Duggan P, Devitt PG, Russell R. The modified essay question: its exit from the exit examination? Med Teach 2010; 32: e300-7.

2. Elementos diagnósticos en medicina interna. URL: http:// campusvirtual.ub.edu/course/view.php?id=17200. [14.05.2011].

3. Moodle. URL: http://docs.moodle.org/es/Tipos_de_preguntas. [14.05.2011].

4. Facultat de Medicina de la Universitat de Barcelona. Competències que han d'adquirir els estudiants de Medicina durant els estudis de pregrau a la Facultat de Medicina de la Universitat de Barcelona. Barcelona: Universitat de Barcelona; 2003.

5. Kronfly E, Delás J, Juncosa S, Blay C, Gràcia LL, Martínez JM. El proyecto 'COMCOR'. Análisis de un método basado en paciente simulados para la evaluación de estudiantes de tercer curso de medicina. Educ Med 2001; 4: 187-93.

6. Delás J, Brasó J, Penzo W. Estudiante-paciente simulado. Educ Med 1999; 2: 129.

7. Ware J, Vik T. Quality assurance of item writing: during the introduction of multiple choice questions in medicine for high stakes examinations. Med Teach 2008; 29: 1-6.

8. Epstein RM. Assessment in medical education. N Engl J Med 2007; 356: 387-96.

9. Case SM, Swanson, DB. Extended-matching items: a practical alternative to free-response questions. Teach Learn Med 1993; 5: 107-15.

10. Palés-Argullós J. ¿Cómo elaborar correctamente preguntas de elección múltiple? Educ Med 2010; 13: 145.

11. Sarrias-Ramis R, Mateu L, Baillès J, Pérez J. Longitud (número de preguntas) y resultado de un examen. Educ Med 2010; 13: 187 . 